\title{
The Evaluation Model and its Application to University Laboratory Safety Based on BP Neural Network \\ Yu Chen ${ }^{1}$, Xiao-guo Liu $^{1}$ \\ ${ }^{1}$ jilin Agricultural University, Changchun, 130118, China
}

\begin{abstract}
Keywords: University laboratory, Laboratory safety, BP neural network, Evaluation model, Evaluation index.
\end{abstract}

\begin{abstract}
The evaluation model of university laboratory safety based on BP neural network is presented. By reference of the domestic research for laboratory safety evaluation and combining with of laboratory characteristics of Jinlin University, the safety evaluation index system of university laboratory is selected. This model quantifies the evaluation index of laboratory safety into definite data as the input for BP network, and taking it output as the evaluation results. The BP network is trained by Trainlm function with training samples which have been obtained by experts, and then it is simulated with testing data. The experiments show that the error between the training value of network simulation and the actual evaluation results is very small, then the applicability of the evaluation model is illustrated. The existing information of laboratory safety evaluation with relatively successful cases can be used in the model, hence the experience of experts can be accumulated for providing more scientific and quantitative criteria for the laboratory safety evaluation.
\end{abstract}

\section{Introduction}

Laboratory safety is an important part of the construction and management of laboratory, has become an important content for the healthy development of science. At present, university laboratory safety hazards everywhere ${ }^{[1-2]}$, lack of laboratory safety management evaluation standard, the system is not perfect, lack of supervision, strict implementation of ${ }^{[3-5]}$. Because of the safety level of the laboratory, in order to provide constructive improvement plan, effectively eliminate the hidden danger, it is worth studying and discussing the problem of the laboratory management work. The impact of many factors were related to the laboratory safety, random large evaluation personnel subjective understanding of the uncertainty and fuzziness, the traditional evaluation methods is difficult to get accurate and objective result, safety precaution and supervision of the laboratory is not clear, guidance is not comprehensive.

BP neural network can effectively deal with the nonlinear, uncertain or fuzzy relation, and has the ability of learning, memory and self adaptation ${ }^{[6]}$. Therefore, this paper based on absorbing BP neural network evaluation in other areas of experience, the application of safety evaluation in university laboratory, a university laboratory safety evaluation model based on BP neural network. The model can be used to case information security evaluation laboratory previously effective memory more successful, the accumulation of expert evaluation experience, scientific and standardized to provide standards and on the basis of laboratory safety management in Colleges and universities.

\section{The importance and necessity of college laboratory safety evaluation}

\section{The importance}

As the scale of higher education expansion, countries of the laboratory construction investment increased year by year, the laboratory have proliferated, open time extended, the researchers more and liquid, various security hidden danger also will increase. In recent years, due to the equipment aging, improper operation, such as lack of fire cause explosion, fire, resulting in experimental facilities were destroyed, casualties, such as frequent accidents, huge losses. Visible, college laboratory safety hidden trouble, the problem that nots allow to ignore. 


\section{The necessity}

College laboratory safety is the key source management, prevention first, do evaluation in advance. Through safety evaluation, timely find out problems, adopt targeted solutions to improve the weak links and hidden dangers in parts or make up for, nip in the bud. Visible, evaluate the regular safety inspection, forecast, default protection, prevent damage, is urgently needed.

\section{Based on the safety evaluation of university laboratory BP neural network model}

\section{Evaluation index system}

According to the establishment of evaluation index system of university laboratory safety principles ${ }^{[6]}$, index selection should take into account data can be ratio and availability, comprehensiveness and parsimony, orientation and sustainability. Through the research on the existing national university laboratory safety evaluation system, combined with the characteristics of laboratory, design the index as the evaluation index system. In this study, the assumption that the same level index weight of the same. College laboratory safety evaluation index system is divided into two levels, the first level indicators are process index, the secondary index is based on indicators, each indicator according to the score from 0 to 10 . Evaluation grade as excellent, good, fair, poor and bad, which corresponds to the fraction of advantages: excellent[9,10];good[8,9]; medium[6,8];poor[5,6];bad[0,5].

The three layer BP network, the input layer for university laboratory safety evaluation index data, the output layer to the evaluation results, the middle hidden layer.

(1) determine the number of neurons in each layer and model. Safety evaluation index of university laboratory is divided into 8 level indicators, 24 level two indexes. 24 level two indexes as the input of BP neural network, so the number of the input neurons of $n=24$. The output neurons as a result of evaluation, the evaluation result is only 1 , so the output neuron number $m=1$. According to the number of neurons in the hidden layer according to the empirical formula ${ }^{[8]}$ $\mathrm{s}=\sqrt{0.43 \mathrm{mn}+0.12 \mathrm{~m}^{2}+2.54 \mathrm{n}+0.75 \mathrm{~m}+0.35}+0.51$ to calculate the number of hidden neurons was 9. So the model constructed is a $24 * 1 * 9 \mathrm{BP}$ neural network model. As shown in Fig 1.

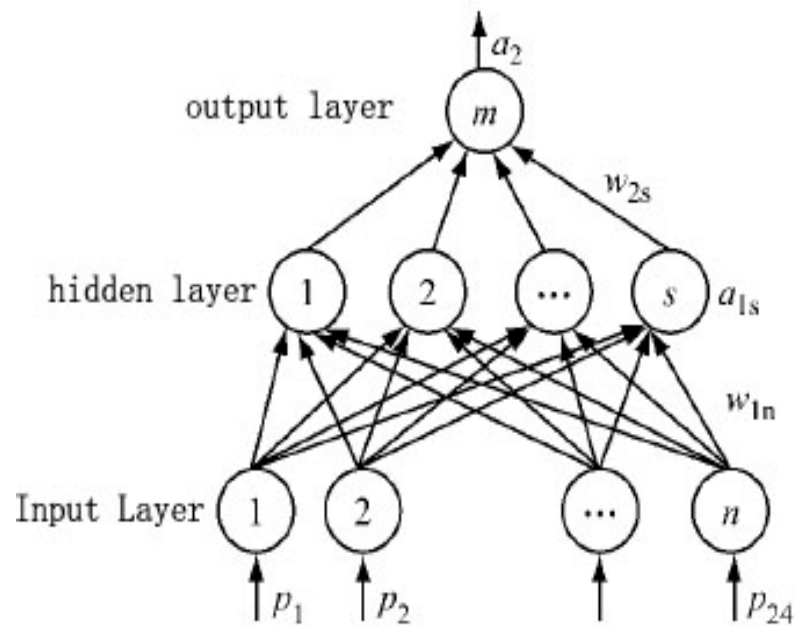

Fig. 1 the structure of BP neural network model for safety evaluation of laboratory

In the network structure, Input vector $\mathrm{P}=\left(\mathrm{p}_{1}, \mathrm{p}_{2}, \cdots, \mathrm{p}_{24}\right)$, The weight of the input layer to the hidden layer is $\mathrm{W}_{1}=\left(\mathrm{w}_{11}, \mathrm{w}_{12}, \cdots, \mathrm{w}_{124}\right)$; Hidden layer output $\mathrm{A}_{1}=\left(\mathrm{a}_{11}, \mathrm{a}_{12}, \cdots, \mathrm{a}_{19}\right)$, The value of the hidden layer to the output layer is $\mathrm{W}_{2}=\left(\mathrm{w}_{21}, \mathrm{w}_{22}, \cdots, \mathrm{w}_{29}\right)$, The actual output of the network is $A=a_{2}=\operatorname{net}\left(A_{1}\right)$.

\section{Evaluation Process}

(1) Data normalization process. Pre-processing the input data, each set of data into the data $[0,1]$, in order to speed up the training of the network. Using normalized formula: $\bar{x}=\frac{x-\operatorname{MIN}(X)}{\max (x)-\min \left(\mathrm{C}_{\mathrm{x}}\right)}$, Be evaluated was obtain the processed input $\left\{\overline{\mathrm{x}}_{\mathrm{i}}\right\}$ 


\section{(2) Evaluation of the step.}

Step 1 network setup and initialization: construct BP neural network by Matlab 7.1 create function newff; as newff creating network objects, while automatic mobilization initialization function in it, according to the default parameters of the network initiates the connection weights and thresholds;

Step 2 network training: the sample data input structure BP neural network training function train through training;

Step 3 Network emulation: to be evaluated test data input trained BP neural network simulation function sim simulation calculated the specific results of the evaluation.

\section{Application examples}

\section{Collection of data samples}

The selection of training samples is the key of network training, the sample selection is appropriate to accumulate experience, network training will be valuable, can be applied to the actual evaluation. China's colleges and universities currently no unified standard of laboratory safety inspection, so this paper in reference to the relevant research results based on, combined with practical work experience, select the evaluation index as the evaluation indexes of the samples, the laboratory of Jinlin University 24 experimental teaching demonstration center are examined, through expert scoring as table 1 shows.

Table 1 Part of the training sample data

\begin{tabular}{|c|c|c|c|c|c|c|c|c|c|c|}
\hline \multirow{2}{*}{ Evaluation } & \multicolumn{6}{|c|}{ Training samples } & \multicolumn{3}{|c|}{ Test Data } & \multirow[b]{2}{*}{$t_{24}$} \\
\hline & $\mathrm{t}_{1}$ & $\mathrm{t}_{2}$ & $\mathrm{t}_{3}$ & $\mathrm{t}_{4}$ & $\mathrm{t}_{5}$ & $t_{6}-t_{20}$ & $t_{21}$ & $t_{22}$ & $t_{23}$ & \\
\hline $\mathrm{P} 1$ & 10 & 10 & 9 & 8 & 9 & $9 \cdots 10$ & 9 & 10 & 10 & 8 \\
\hline P2 & 8 & 9 & 9 & 7 & 8 & $9 \cdots 7$ & 9 & 9 & 9 & 9 \\
\hline P3 & 8 & 9 & 9 & 8 & 8 & $9 \cdots 9$ & 10 & 8 & 8 & 7 \\
\hline $\mathrm{P} 4$ & 9 & 8 & 10 & 7 & 9 & $9 \cdots 8$ & 7 & 9 & 9 & 9 \\
\hline P5 & 8 & 9 & 9 & 8 & 10 & $9 \cdots 10$ & 9 & 8 & 7 & 10 \\
\hline P6 & 9 & 8 & 7 & 9 & 8 & $9 \cdots 7$ & 8 & 9 & 10 & 8 \\
\hline$\cdots$ & $\cdots$ & $\cdots$ & $\cdots$ & $\cdots$ & $\cdots$ & $\cdots \cdots$ & $\cdots$ & $\cdots$ & $\cdots$ & $\cdots$ \\
\hline p24 & $10 \cdots 9$ & $8 \cdots 7$ & $9 \cdots 10$ & $7 \cdots 9$ & $7 \cdots 8$ & $8 \cdots 9$ & $9 \cdots 8$ & $8 \cdots 9$ & $8 \cdots 9$ & $9 \cdots 10$ \\
\hline $\begin{array}{l}\text { Evaluation } \\
\text { results }\end{array}$ & 8.31 & 8.03 & 8.79 & 8.01 & 7.44 & $7.91 \cdots 8.01$ & 9.2 & 8.01 & 8.88 & 8.04 \\
\hline $\begin{array}{l}\text { evaluation } \\
\text { grade }\end{array}$ & good & good & good & good & medium & medium $\cdots$ good & good & good & good & good \\
\hline
\end{tabular}

\section{Network training and simulation}

Using the artificial neural network toolbox of Matlab7.1 software NNbox training network. The number of laboratory safety evaluation index is 24 , so the number of input layer neuron is 24 , the number of output layer neuron is 1 , and the number of hidden layer is 9. Setting the training function is trainlm, hidden layer transfer function is Tansig function, the output layer transfer function is purelin. The maximum number of training for 1000, target error is less than 0.001 , learning efficiency is 0.1 , weights and thresholds to take a random number, reached 12 times when the number of training, target error is less than 0.001, network training ended.

After the end of the training, the SIM function is used to simulate the simulation. The last 4 groups of test samples were brought into the trained network to evaluate the network simulation results as shown in Table2. In Matlab7.1, the network simulation training value and the actual evaluation results as the 2 series of graphics output, get the contrast curve shown in figure 2 . 
Table 2 Network simulation results of the test sample data

\begin{tabular}{cccc}
\hline Test data & Test & Network1 outputs & Network1 errors \\
\hline $\mathbf{T}^{21}$ & 8.57 & 8.681 & -0.020 \\
$\mathbf{T}^{22}$ & 8.88 & 8.875 & -0.015 \\
$\mathbf{T}^{23}$ & 7.51 & 7.531 & -0.001 \\
$\mathbf{T}^{24}$ & 9.03 & 8.970 & 0.066 \\
\hline
\end{tabular}

\section{Result analysis}

From the Table 2 and Figure 2, we can see that after the training of neural network output of the training value and the actual evaluation of the error is very small, verify the accuracy and rationality of the evaluation model. But because there is a subjective assessment of the evaluation of the main error, evaluation factors of the coverage is not comprehensive enough, sample size smaller, BP nerve network oneself also some limitations, the evaluation results are inevitable existence some error.

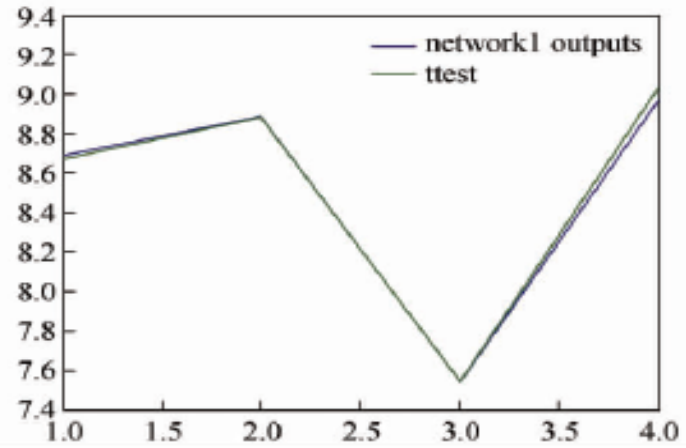

Fig 2. Network simulation training value and the actual results of the evaluation the comparison chart

\section{Conclusion}

In summary, college laboratory safety evaluation model based on BP neural network has the following advantages: (1) The model is a good way to learn from experts experience, use of the existing evaluation results, accumulated expertise, and preserved by evaluation data and is applied to the actual evaluation work. (2) The model also has wide applicability, each university may, in accordance with its own characteristics for teaching experiment to changes of index system, at the same time can also influence the degree of different indicators gives different weights to apply in order to achieve results. (3) Using the Matlab neural network toolbox, solving method is simple, all calculations are performed by computer, solved the problem of the other methods computational burden, easy to use, easy to promote. Aiming at evaluation results the existence of some error in the experiment, the future will also to improve the model, make it more reasonable, the evaluation results more accurate.

\section{References}

[1] Ruan Jun, Discussion on the inspection and rectification of potential safety hazard in the management of laboratories in colleges and universities [J].Experimental Technology and Management, 2010,27(9):190-192.

[2] Chen Lijun. Study and Exploration on Management System of University Laboratory [J]. Research and Exploration in Laboratory, 2010, 29(7):339-341.

[3] Wang Jie. Discussion on laboratory safety management and study of its countermeasures [J] Experimental Technology and Management 2010, 27(3):247-252. 
[4] Yu jianxin.et.al. Model and Application of Safety Evaluation for Campus Based on BP-neural Network [J]. Safety and Environmental Engineering,,2011,18(2):93-95.

[5] Kranz M. Embedded interaction: Interacting with the internet of things [J]. IEEE Internet Computing, 2010, 14( 2) : 46-53.

[6] Alphand, O. Duda, A. Heusse, M. Ponsard, B. Rousseau, F. Theoleyre, F. Towards the Future Internet of Sensors[C]//The Internet of Things 2010, doi: 10.1007/978-1 -4419-1674- 7_30.

[8] Daniele Miorandi, Sabrina Sicari, Francesco De Pellegrini, et al. Internet of things: Vision, applications and researchchallenges[J]. Ad Hoc Networks, 2012 ( 10 ) : 1497-1516. 